\title{
Traitement de la streptothricose bovine par une injection unique d'antibiotiques à haute dose
}

\author{
par J. M. BLANCOU
}

\begin{abstract}
RÉSUMÉ
Une injection intramusculaıre unique de Pénıcilline $(75.000$ U. $1 . / \mathrm{Kg}$ de poids vif) associée à la Streptomycine ( $75 \mathrm{mmg}^{\prime} \mathrm{kg}$ de poids vif) guérit en 15 jours des bovıns atteınts de Streptothricose grave, dans 70 p. 100 à 90 p. 100 des cas.
\end{abstract}

Les antibiotıques ont été utilisés par de nombreux auteurs dans le traitement de la Streptothricose des ruminants, par voie parentérale, avec des résultats très différents. Certains leur accordent une efficacité réelle (1.2.12 13) d'autres une efficacité très passagère ou nulle $(4.5 .7)$.

L'usage de ces mêmes antibiotiques a été préconisé avec succès dans certaınes maladies dont l'agent causal est envahissant ou protégé par une réaction tissularre de l'organisme : septicémies, endocardites, affections cutanées, « Lumpy wool disease » en particulier. II nous a paru logique de tenter un nouveau tratement antibiotique de la Streptothricose bovine avec une posologie différente, décuplée par rapport à la posologie classique.

Nous exposons ici les résultats obtenus par l'administration d'une association de Pénicillıne et de Streptomycine à haute dose. L'activité particulière de ces deux antibiotıques « in vitro» sur Dermatophilus Congolensis, et « in vivo » chez les ovins (10.12.14), leur absence de toxicité aux doses élevées et leur faıble prix de revient nous ont guidé dans le choix de cette association.

Le falt qu'à Madagascar, les animaux de races importées (Brahman, Limousın, Normand) et leurs croisements solent les plus gravement atteints (2.14) justıfie le coût de l'intervention : 300 à 400 Francs malgaches par sujet environ.

TABLEAU $\mathrm{N}^{*} \mathrm{I}$

Caractéristiques des animaux d'expérience

\begin{tabular}{|c|c|c|c|c|c|}
\hline \multirow[b]{2}{*}{$\begin{array}{l}\text { Anlmaux } \\
\text { traitês }\end{array}$} & $\mathrm{R} a \mathrm{c}$ & \multirow{2}{*}{ 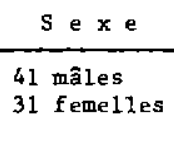 } & \multirow{2}{*}{ 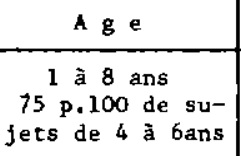 } & \multicolumn{2}{|c|}{ Degré d'atteinte } \\
\hline & $\begin{array}{l}\text { Zébu malgache crolsê } \\
\text { de zébu Brahman }\end{array}$ & & & $\begin{array}{r}+++ \\
++ \\
+\end{array}$ & $\begin{array}{r}3 \\
44 \\
25\end{array}$ \\
\hline $\begin{array}{l}\text { Anlmaux } \\
\text { têmolns }\end{array}$ & $\begin{array}{c}\text { Zébu malgache croisé } \\
\text { de zébu Brahman }\end{array}$ & $\begin{array}{l}45 \text { mâles } \\
25 \text { femelles }\end{array}$ & $\begin{array}{c}1 \text { à } 8 \text { ans } \\
75 \text { p. } 100 \text { de su- } \\
\text { jets de } 4 \text { à } 6 \text { ans }\end{array}$ & $\begin{array}{r}+++ \\
++ \\
+\end{array}$ & $\begin{array}{r}2 \\
38 \\
30\end{array}$ \\
\hline
\end{tabular}

N.B. +++ = atteinte gênéralisée; ++ = atteinte de plus de la moitié de la surface cutanée ; + attelnte de plus du quart de lo surface cutanée. 


\section{MODALITÉS DU TRAITEMENT}

\section{Animaux d'expérience.}

II s'agissait d'animaux atteints de Streptothricose naturelle, vivant en élevage extensif. Les caractéristiques principales des différents sujets d'expériences sont indıquées dans le tableau I :

\section{Antibiotiques utilisés, posologie, mode d'admi- nistration.}

Nous avons utilisé les antibiotiques usuels du commerce sous la forme de BipénicillineDidromycine associés selon la formule suivante :

Dihydrostreptomycine base (sul-

fate) .................

Benzylpénıcıllinate de sodium cris-

tallısé ................. 400.000 UI

Benzylpénıcıllınate de procaîne lé-

cuthiné .................. 600.000 UI

La posologie adoptée, après essais préliminarres, est de $75 \mathrm{mg}$ de Streptomycıne associés à 75.000 Ul de Pénicilline par kilogramme de poids vif. La solution est admınıstrée en une seule injection intra-musculaire au niveau de l'encolure. Après l'injection, nous n'avons jamais observé d'accident immédıat ou retardé.

\section{Contrôle des résultats.}

Deux critères ont été adoptés pour apprécier l'évolution de la maladie avant et après traitement :

1. - Examen clinique complet : état général, poids, état de la peau.

2. - Valeur du rapport Albumıne/Globulınes sérıques.

Deux difficultés sont à soulıgner dans l'interprétation des résultats :

- L'intervention de facteurs incontrôlables en élevage extensif : climat, alimentation, traitements antıparasitaires collectıfs. Pour cela, un cholx rigoureux de sujets témoins est indispensable.

- L'éventualıté de différences d'un troupeau à l'autre, telles qu'elles apparaissent dans les expériences de C. B. HART et coll. (6) opérant sur des effectifs importants d'ovins. Dans le cas des bovins, des traitements de sondage sur d'autres troupeaux semblent infirmer cette possibılıté. Mais seule une expérience étendue du traltement permettra de s'en assurer.

\section{RÉSULTATS}

\section{Examen clinique.}

Il est pratiqué quinze jours après le traitement, puis contrôlé régulièrement jusqu'au cinquième mols, où le résultat définitif est consigné.

1. - Lot de 70 animaux fémorns : ce lot comprenait des sujets de même sexe et âge que le lot des animaux trattés, observés durant la même période.

Observation au cinquième mois :

Etat aggravé ................. 3

Etat stationnare ............... 56

Ełat amélioré .................. 8

Guérıson totale ................. 3

2. - Lot de 72 animaux traités:

Etat aggravé ................. 1

Etat stationnaire ................. 3

Etat amélıoré ................... 10

Guérison totale ................ 58

Dans la majorité des cas la guérison totale est obtenue dès le $15 \mathrm{e}$ jour. Parmi les 14 sujets n'ayant pas guérl totalement après un mols, 7 ont été soumis à un nouveau traltement, aux mêmes doses : 4 d'entre eux ont guéri totalement en 15 jours, les 3 autres sont restés incurables.

\section{Valeur du rapport albumine/globulines séri- ques $(*)$.}

La valeur de ce rapport reflète toujours fidèlement l'état clinique du sujet (14). A titre Indicatif, voici quelques valeurs relevées au hasard chez des animaux témoins et des animaux trattés, à un mols d'intervalle.

1. - Chez dix malades non traités :

Février : $\begin{array}{lllllllll}0,30 & 0,33 & 0,11 & 0,35 & 0,30 & 0,37 & 0,20\end{array}$ $0,330,200,20$.

Moyenne : 0,27 .

(*) Analyses effectuées au Laboratorre Central de I'Elevage de Tananarive par R. GAULIER, que nous remercions vivement. 
Mars (état stationnaire) : $0,27 \quad 0,33 \quad 0,10 \quad 0,41$

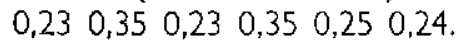

$$
\text { Moyenne : } 0,28 \text {. }
$$

2. - Chez dix malades traités :

Février (Traitement) : $0,33 \quad 0,150,350,33$

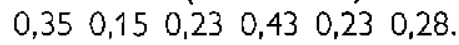

Moyenne : 0,28 .

Mars (Guérison) : $0,47 \quad 0,27 \quad 0,450,41 \quad 0,45$ $\begin{array}{lllll}0,27 & 0,35 & 0,54 & 0,45 & 0,45\end{array}$.

$$
\text { Moyenne : } 0,41 \text {. }
$$

La différence des moyennes $(0,01$ chez les témoins contre 0,13 chez les animaux traltés) témoigne d'une importante amélioration de l'état général.

\section{CONCLUSION}

Les résultats précédents, étudiés selon la méthode statistique du $\chi^{2}$ corrigé pour la contnuité démontrent une action hautement sıgnıficatıve du traitement sur l'évolutıon de la maladıe. On peut espérer (coefficient de confiance $95 \mathrm{p} .100$ ) la guérison totale de 70 p. 100 à 90 p. 100 des malades en une seule intervention.

\section{DISCUSSION}

L'étude sérologique, par la méthode de I'hémagglutination passive (8.9.14) nous a démontré une augmentation significative du taux des anticorps après traitement. Cette observatıon est à rapprocher du fait que les animaux réfractaires au traitement étalent en général plus jeunes que les autres, et qu'ils guérissent parfors lors d'un second traitement. Cet ensemble de constatations suggère l'intervention possible d'un phénomène immunologıque renforçant l'action des antibiotıques : la lyse brutale du Dermatophilus Congolensis provoquant une réacfion anamnestique de l'organısme dont les antıcorps auraient alors une activité bactérıstatique.

Une vaccination du type de celles tentées précédemment chez les bovins (9.13) aurait probablement une efficacité accrue chez de tels animaux. L'antibiothérapie est cependant un traitement individuel. Le traitement collectif reste onéreux et perdralt rapidement de son efficacité au cours de grandes épizooties où trop d'ındıvidus se réinfectent et où peuvent apparaître de soudaines antıbiorésistances.

Laboratorre Central de l'Elevage. Tonanarive.

\section{REMERCIEMENTS}

Nous tenons à remercier Messieurs GILIBERT J., de REVIERS B. et TARDIF J. du C. R. Z. F. de KIANJASOA pour le concours qu'ils nous ont apporté lors de ces traitements expérimentaux.

\section{SUMMARY \\ Treatment of bovine sireptothricosis by only an high dose antibiotic injection}

Cattle caught by serious streptothricosis are, in $70 \mathrm{p} 100$ to $90 \mathrm{p} 100$ of cases, cured in 15 days by only a muscular injection of Penicilitin $(75000 \mathrm{U} .1 . / \mathrm{kg}$ of live weight) associated with streptomycin (75 mmg kg of live waight).

\section{RESUMEN}

Trałamiento de la estraptotricosis de los bovinos madiante una inyección únıca de antibiolicos en dosis elevada.

Una inyección intramuscular única de penicilina (75000 U likg de peso vivo) asociada con la estreptomicina ( $75 \mathrm{mmg} / \mathrm{kg}$ de peso vivo) cura a los 15 dias bovinos atacados por estreplotricosis grave en 70 a 90 p. 100 de los casos. 


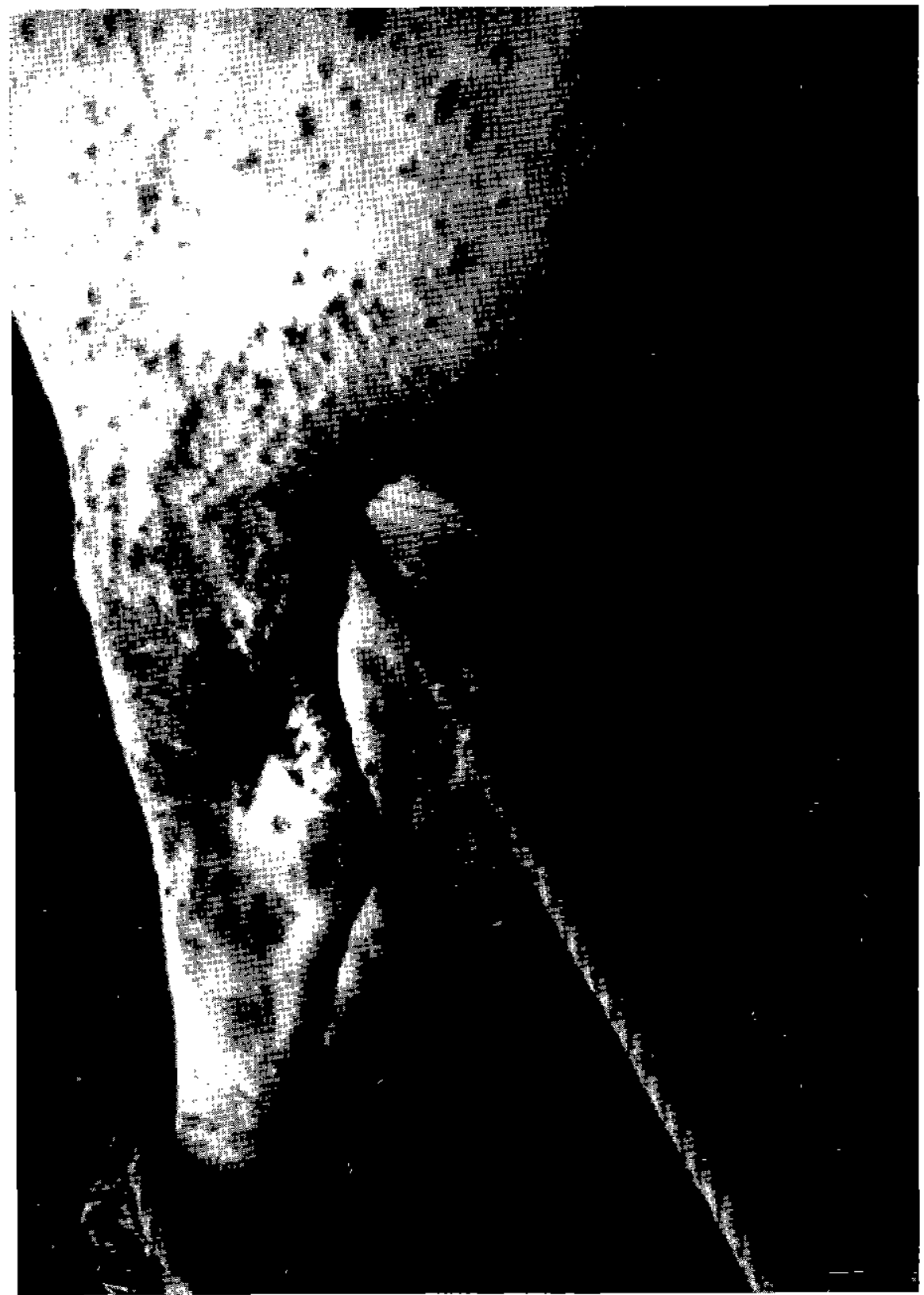

Photo no 1. - Taureau 3/4 Brahmon atteınt de Streptothrıcose généralisée.

Traté le 11 avril 1968 par 35 millions U. I. de Péntcıllıne et 35 grammes de Streptomycine. 


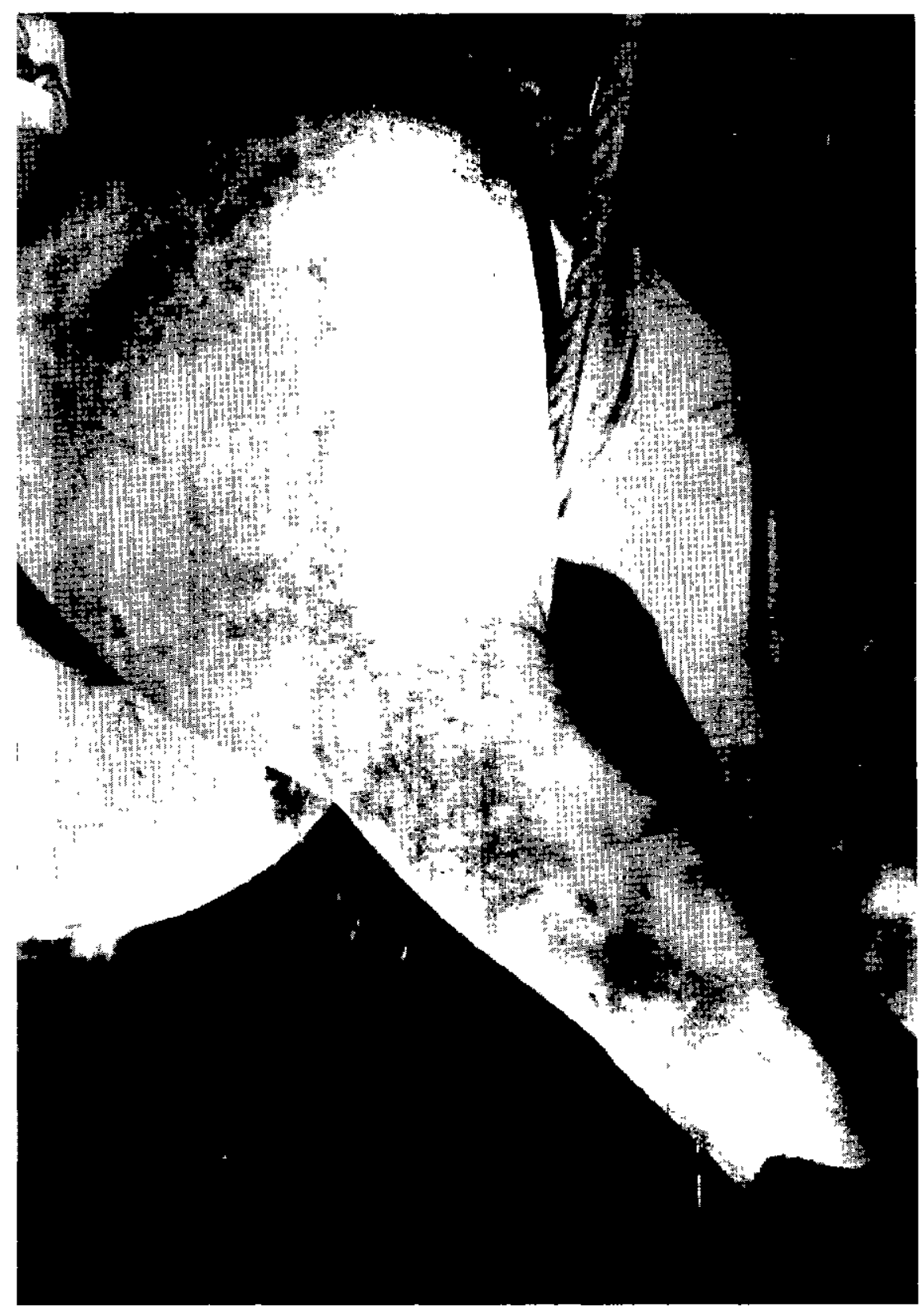

Photo $n^{\circ} 2$ - Même anımal le 21 avrıl. 


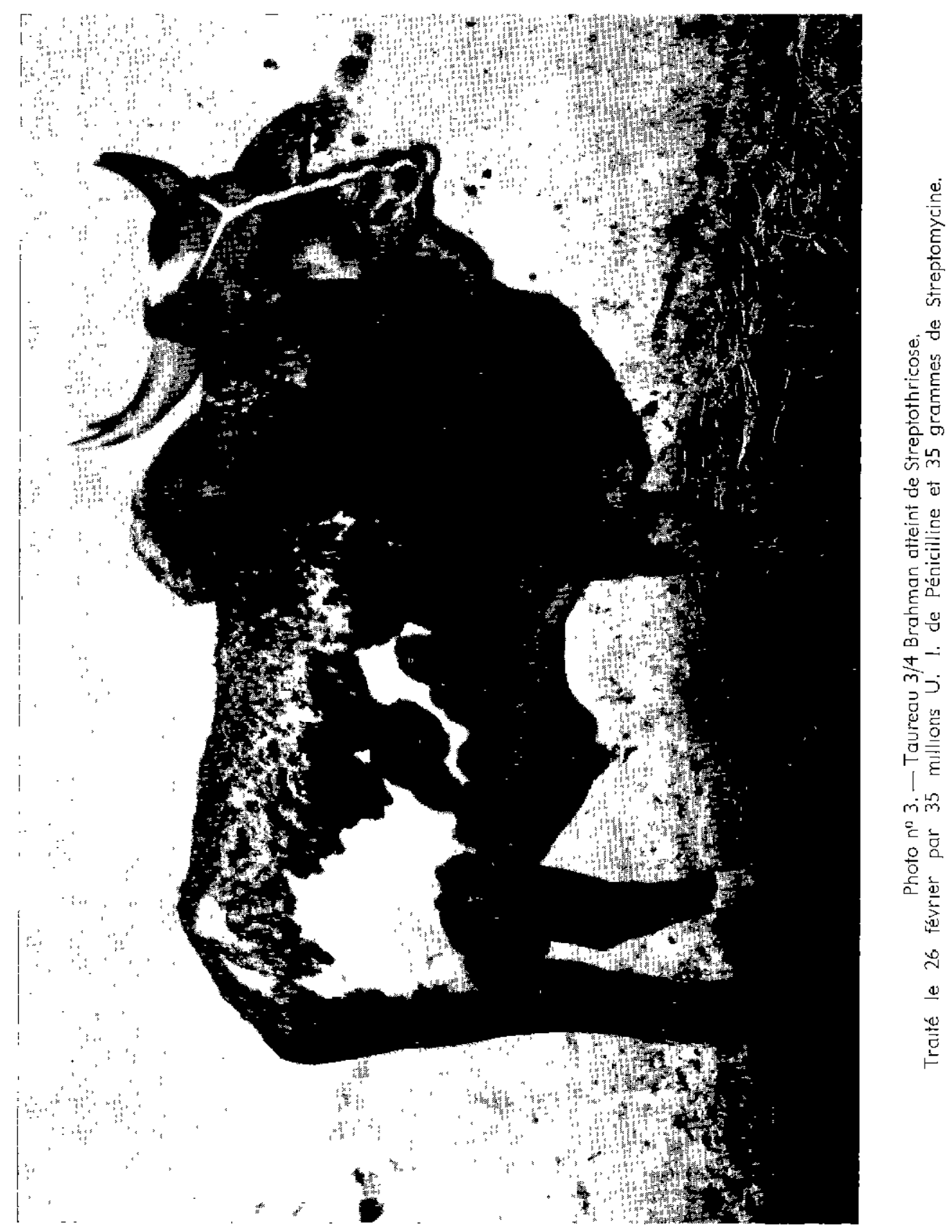




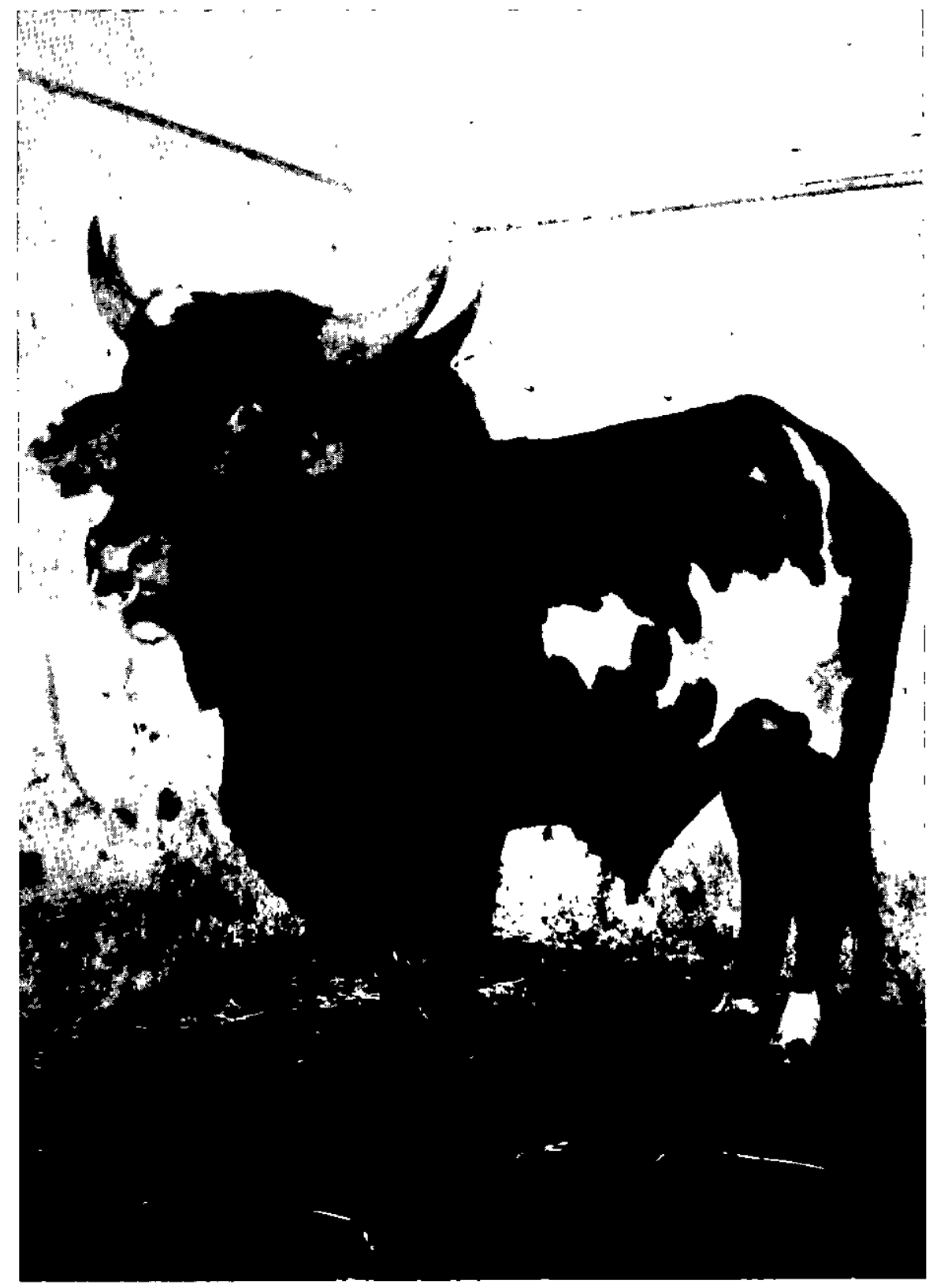

Photo $n^{\circ} 4$ - Même anirraí le 11 mars 


\section{BIBLIOGRAPHIE \\ (Références citées)}

1. ANONYME. - Seventh annual report of the Commonwealth Scientific and industrial Research organisation for the year, ending 30th June 1955, 1955, p. 185.

2. BUCK (G.). - Actinomycose ou Streptothricose cutanée des bovins à Madagascar. Bull. Off. Int. Epiz., 1948, 29 (3-4), 117-121.

3. BUGYAKI (L.). - Dermatose contagieuse des ruminants et du cheval (Streptothricose - Actimomycose cutanée). Bull. Off. int. Epiz., 1959, 51 (5-6), 237-249.

4. COLEMAN (C. H.). - Cutaneous streptothricosis of cattle in West Africa. Vel. Rec., 1967, 81 (11), 252-254.

5. EGERTON (J. R.). - Mycotitic dermatitis of cattle. Austr. Vet. J., 1964, 40, 144-147.

6. HART (C. B.), TYSZKIEWICZ (K.). Mycotic dermatitis in sheep. Parl III. Chemotherapy with Potassium Aluminium sulphate. Vet. Rec., 1968, 82 (10).

7. MAMMERICKX (M.). - Observations sur la dermatose contagieuse des ruminants au Congo. Ann. Soc. Beige Med. Trop., 1961, 41, 133-144.
8. PULLIAM (」. D.), KELLEY (D. C.), COLES (E. H.) - Immunologic studies of natural and experimental Streptothricosis infection in cattle. Am. J. Vet. Res., 1967, 28 (123), 447-55.

9. PERREAU (P.), CHAMBRON (J.). - Immunologie de la Streptothricose cutanée des bovins. Essais de vaccination. Rev. Elev. Méd. vét. Poys trop., 1966, 19 (3), 263-74.

19. ROBERTS (D. S.). - Dermatophilus infection. Vet. Bull., 1967, 37 (8), 513-21.

11. ROBERTS (D. S.), - Chemotherapy of epidermal infection with Dermatophilus Congolensis. J. Comp. Path., 1967, 77 (2), 129-36.

12. THIERY (G.), MEMERY (G.). - Lo Streptothricose cutanée. IV. Etiologie, traitement, prophylaxie. Rev. Elev. Méd. Vét. Pays trop., 1961,14 (4), 415-27.

13. Rapport annuel 1967 de la Région de Recherches Vétérinaires et Zootechniques de I'I.E.M.V.T. en Afrique Centrale, Tome II. Streptothricose bovine. Bilan d'actıyité.

14. Rapports annuels de la Région de Recherches de I'I. E. M. V. T. à Madagascar. 